\title{
Potential Metabolite Markers for Pancreatic Cancer Identified by Metabolomic Analysis of Induced Cancer-Associated
} Fibroblasts

\author{
Yoshihiro Miyazaki ${ }^{1,2}$, Nobuhito Mori' ${ }^{2}$, Yuka Akagi' ${ }^{2}$, Tatsuya Oda ${ }^{1}$ and Yasuyuki S. Kidaa ${ }^{2,3,4^{*}}$
}

\author{
${ }^{1}$ Department of Gastrointestinal and Hepato-Biliary-Pancreatic Surgery, Faculty of Medicine, University of \\ Tsukuba, Tsukuba, Japan \\ ${ }^{2}$ Cellular and Molecular Biotechnology Research Institute, National Institute of Advanced Industrial Science \\ and Technology (AIST), Tsukuba, Japan. \\ ${ }^{3}$ Advanced Photonics and Biosensing Open Innovation Laboratory, The National Institute of Advanced \\ Industrial Science and Technology (AIST), Tsukuba, Japan. \\ ${ }^{4}$ School of Integrative and Global Majors, University of Tsukuba, Tsukuba, Japan. \\ * Correspondence: Yasuyuki S. Kida \\ Cellular and Molecular Biotechnology Research Institute, National Institute of Advanced Industrial Science \\ and Technology (AIST), Central 5-41, Higashi 1-1-1, Tsukuba 305-8565 Ibaraki, Japan. \\ Tel: +81-29-861-3000 \\ Fax: $+81-29-861-3000$ \\ Email: y-kida@aist.go.jp
}

Simple summary: Fibroblasts in normal tissues conduct energy metabolism via oxidative phosphorylation (OXPHOS). However, cancer-associated fibroblasts (CAFs) produce energy (i.e., ATP) via glycolysis. Nonetheless, whether intracellular metabolism transitions from OXPHOS to glycolysis when normal tissue fibroblasts differentiate into CAFs remains to be determined. Here, we established an experimental system and induced the in vitro differentiation of mesenchymal stem cells to CAFs and performed detailed metabolomic and RNA sequencing analyses. We found that the intracellular metabolic pathway was reprogrammed to the glycolytic pathway when mesenchymal stem cells were co-cultured with pancreatic cancer cells. Furthermore, we identified CAF-specific metabolites that were expressed post reprogramming. These metabolites have also been observed in pancreatic cancer mouse models, suggesting their potential as cancer biomarkers.

\begin{abstract}
Cancer-associated fibroblasts (CAFs) in the tumor microenvironment perform glycolysis to produce energy, i.e., ATP. Since the origin of CAFs is unidentified, it is not determined whether the intracellular metabolism transitions from oxidative phosphorylation (OXPHOS) to glycolysis when normal tissue fibroblasts differentiate into CAFs. In this study, we established an experimental system and induced the in vitro differentiation of mesenchymal stem cells (MSCs) to CAFs. Additionally, we performed metabolomic and RNA-sequencing analyses before and after differentiation to investigate changes in the intracellular metabolism. Consequently, we discovered that OXPHOS, which was the primary intracellular metabolism in MSCs, was reprogrammed to glycolysis. In addition, we identified CAF-specific metabolites that were expressed during this reprogramming and determined their presence in the pancreatic tumor tissues of mouse models. Thus, we conclude that normal tissue fibroblasts that differentiate into CAFs undergo a metabolic reprogramming from OXPHOS to glycolysis. Moreover, we identified the CAF-specific metabolites expressed during metabolic reprogramming as potential future biomarkers for pancreatic cancer.
\end{abstract}

Keywords: cancer-associated fibroblasts; tumor microenvironment; pancreatic cancer; intracellular metabolism; glycolysis; oxidative phosphorylation; cell differentiation 


\section{Introduction}

The most common solid tumors, such as pancreatic cancer, have a large number of stromal cells. Indeed, the stroma forms a thickened extracellular matrix that provides a unique environment, such as a hypoxic environment by inhibiting blood vessel formation in a tumor. The thickened extracellular matrix acts as a barrier that protects cancer cells from attack by the immune system and anticancer drugs, drastically reducing the response rate of patients to cancer therapy [1-3].

The tumor microenvironment (TME) comprises cancer cells surrounded by a variety of stromal cells, including cancer-associated fibroblasts (CAFs), tumor-associated macrophages, and infiltrating immune cells. These stromal cells interact with cytokines and metabolites and create an environment that promotes the aggressive growth of cancer cells $[4,5]$.

Notably, CAFs are the most abundant cells in the TME. Studies have demonstrated that CAFs are produced upon the epithelial-to-mesenchymal transition of cancer cells and are derived from fibroblasts localized in tissues [6,7] and mesenchymal stem cells (MSCs) derived from the bone marrow [8] or adipose tissue $[9,10]$. However, their origin has not been determined in human tumors despite their presence being determined in tumorigenesis experiments in vivo. In fact, most CAFs in the TME do not have the genetic mutations that are typically observed in cancer cells. Recently, researchers have expressed increasing interest in how normal cells, such as MSCs, adapt to the TME and differentiate into CAFs that support carcinogenesis; indeed, much research has been done to elucidate this mechanism [11]. Drug discovery-based research targeting the inhibition of CAFmediated stromal support to kill cancer cells has also been attracting attention [12].

Hypoxia and low nutrient supply make the TME an unfavorable environment for cell proliferation. Interestingly, cancer cells overcome this disadvantage by expressing hypoxia-responsive genes, thereby proliferating rapidly [13]. Moreover, they counteract this unfavorable environment of the TME by maintaining a special intracellular metabolic state called the Warburg effect, and also owing to powerful genetic mutations, such as those in Kras and p53 [14,15]. However, how CAFs survive adverse conditions, such as hypoxia and low nutrient supply, in the TME has not yet been well studied. Inhibition of several gene functions in CAFs has suggested that glycolysis is upregulated in CAF, thereby increasing ATP production. Moreover, CAFs have been demonstrated to release metabolites that are necessary for the survival and functioning of cancer cells [16,17]. This is called the reverse Warburg effect and is considered to be one of the primary mechanisms through which CAFs propagate cancer cell survival. However, metabolic shifts in a normal cell, such as the upregulation of glycolysis and downregulation of oxidative phosphorylation (OXPHOS), has not been studied yet. This is because progenitor cells of CAFs are as yet unidentified, thereby making it difficult to experimentally induce cell differentiation to CAFs.

Previously, we have demonstrated that adipose-derived MSCs (AD-MSCs) can differentiate into CAFs in vitro and in vivo and can reproduce a robust TME that is frequently observed in pancreatic cancer [10] [18]. These studies revealed that AD-MSCs are progenitors of CAFs; they morphologically transform into CAFs, exhibit alterations in their gene expression, and acquire heterogeneity. In the present study, we performed in vitro metabolomic analysis in induced CAFs to understand the metabolic shift that takes place in CAFs. We discovered a prominent metabolic shift towards glycolysis accompanied with downregulated OXPHOS and identified novel metabolites that were produced during cell differentiation to CAFs. 


\section{Materials and Methods}

\subsection{Cells and culture conditions}

The immortalized human AD-MSC cell line ASC52telo (ATCC SCRC-4000) and the human pancreatic cancer cell line Capan-1 (ATCC HTB-79) were used and cultured as previously described [10] [18]. Briefly, these cells were maintained in Dulbecco's Modified Eagle's Medium (DMEM; FUJIFILM Wako Pure Chemical Corp., Osaka, Japan) supplemented with $20 \%$ fetal bovine serum (FBS), $1 \%$ nonessential amino acids, and $1 \%$ streptomycin-penicillin at $37{ }^{\circ} \mathrm{C}$ in a humidified atmosphere containing $5 \% \mathrm{CO}_{2}$. Additionally, the KPC cell line derived from the KPC mice was maintained in DMEM supplemented with $10 \%$ FBS and $1 \%$ streptomycin-penicillin at $37{ }^{\circ} \mathrm{C}$ in a humidified atmosphere containing 5\% $\mathrm{CO}_{2}$. Subsequently, the AD-MSCs and Capan-1 cells were labeled with green fluorescent protein (GFP) and red fluorescent protein (RFP) by lentiviral transduction, respectively. For this purpose, we seeded 293LTV cells (LTV-100; Cell Billabs, Inc.) into a six-well plate and co-transfected them with pLKO.1-puro eGFP (Sigma-Aldrich, St. Louis, MO, USA), psPAX2 (\#12260; addgene), and pMD2.G (\#12259; addgene) at a ratio of 2:1.5:1.2 $\mu \mathrm{g} /$ well. Post $24 \mathrm{~h}$, the viral supernatant was collected and filtered through $0.45 \mu \mathrm{m}$ membranes. Thereafter, AD-MSCs were infected with this filtrate in the presence of polybrene $(10 \mu \mathrm{g} / \mathrm{mL})$ for $24 \mathrm{~h}$. Thereafter, puromycin was added to the medium for the selection of GFP-positive cells. Once we identified AD-MSCs that expressed GFPs, we confirmed that these cells still retained their original characteristics, such as auto-differentiation, senescence, or weak stemness.

\subsection{In vitro co-culture assay}

Co-culturing of AD-MSCs and Capan-1 cells was performed as described previously [18]. In this regard, AD-MSCs and Capan-1 cells were co-cultured under two different conditions: direct and indirect transwell co-culture. In the direct co-culture method, $4 \times$ $10^{5}$ AD-MSCs and $4 \times 10^{5}$ Capan- 1 cells were mixed and seeded in six-well culture plates. In contrast, in indirect transwell co-culture, $4 \times 10^{5} \mathrm{AD}-\mathrm{MSC}$ sere seeded in the lower compartment of the transwell membrane, whereas $4 \times 10^{5}$ Capan- 1 cells were seeded in the upper compartment (Falcon Permeable Support for 6-well plates with 3.0- $\mu$ m translucent high-density PET membrane \#353092; Corning, Corning, NY, USA).

\subsection{Immunofluorescence (IF) staining of cells in monocultures and co-cultures}

We fixed AD-MSCs and Capan-1 cells with $100 \%$ methanol for $10 \mathrm{~min}$ at $-20{ }^{\circ} \mathrm{C}$. Subsequently, we washed them thrice for 5 min each with an IF buffer solution $(10 \times$ stock: $38.0 \mathrm{~g} \mathrm{NaCl}, 9.38 \mathrm{~g} \mathrm{Na}_{2} \mathrm{HPO}_{4}, 2.07 \mathrm{~g} \mathrm{NaH}_{2} \mathrm{PO}_{4}, 2.5 \mathrm{~g} \mathrm{NaN}_{3}, 5.0 \mathrm{~g}$ bovine serum albumin (BSA), $10 \mathrm{~mL}$ Triton X-100, and $2.5 \mathrm{~mL}$ Tween-20 in $500 \mathrm{~mL}$ PBS). Thereafter, the cells were treated with a blocking solution ( $3 \%$ BSA in $1 \times$ IF washing solution) for $30 \mathrm{~min}$. They were then incubated for $1 \mathrm{~h}$ at $25{ }^{\circ} \mathrm{C}$ with a mouse SMA antibody (1: 400, ab7817; Abcam, Cambridge, UK), rabbit anti IL-6 antibody (1: 200, ab6672; Abcam), or rabbit anti rat vimentin antibody (1: 400, \#280618; R\&D Systems Inc., Minneapolis, MN, USA) in the $1 \times$ IF buffer. Post this incubation, the cells were washed thrice with the $1 \times$ IF buffer. They were subsequently incubated for $1 \mathrm{~h}$ with Alexa Fluor 488 goat anti-rabbit IgG antibody (Invitrogen, Carlsbad, CA, USA), Alexa Fluor 568 goat anti-rat IgG antibody (Invitrogen), and Cy5 goat anti-rabbit IgG antibody (Invitrogen), all diluted to 1: 400 in the $1 \times$ IF buffer solution. The cell nuclei were counterstained with Hoechst 33342 (Thermo Fisher Scientific, Waltham, MA, USA) and thereafter were washed thrice with the $1 \times$ IF buffer. The slides were mounted and visualized under a fluorescence microscope [BZ-710, Keyence, Osaka, Japan]).

\subsection{In vitro macropinocytosis assay}

We performed an in vitro macropinocytosis assay as previously described [19]. Briefly, $1 \times 10^{5}$ cells were plated onto glass coverslips in six-well plates for five days. We then incubated the cells for $30 \mathrm{~min}$ at $37^{\circ} \mathrm{C}$ with $70-\mathrm{kDa}$ FITC-dextran (Sigma) directly added to the culture media at a final concentration of $1 \mathrm{mg} / \mathrm{mL}$. Subsequently, we assessed the macropinocytic uptake of cells, and rinsed the cells five times on ice with ice-cold PBS. 
Thereafter, the cells were fixed with $3.7 \%$ formaldehyde and their nuclei were counterstained with DAPI. Eventually, the coverslips were mounted onto glass slides using an aqua-poly/mount (Polysciences, Inc.). Fluorescent images were captured using a fluorescence microscope (BZ-710; Keyence). Notably, each experimental condition was performed in triplicates.

\subsection{Mouse models and in vivo experiments}

We purchased eight-week-old female C57BL/6J wild mice from Japan CLEA Inc. (CLEA Japan, Tokyo, Japan) for our experiments. We bred and housed the mice under specific pathogen-free conditions at the Animal Center of AIST and the University of Tsukuba. We developed a xenograft model by subcutaneously transplanting $2 \times 10^{6} \mathrm{KPC}$ cells into the mice (KPC xenograft model). Thereafter, we sacrificed the mice on day 28 and excised all subcutaneous tumors.

All invasive procedures were performed under inhalation anesthesia using isoflurane. Mice were euthanized by cervical dislocation following inhalation of the anesthesia. All animal experiments and procedures were approved by and performed in compliance with the guidelines of Institutional Animal Care and Use Committee of the respective institutes of AIST (A2020-310) and the Ethics Committee of the University of Tsukuba (19-028). The study was conducted in accordance with the Animal Research Reporting in vivo Experiments (ARRIVE) guidelines [20].

\subsection{Immunohistochemical tissue staining}

All the staining protocols were performed on $2 \mu \mathrm{m}$-thick mouse tissue sections. Hematoxylin and eosin (HE) and Masson's trichrome (MT) staining were performed according to standard protocols [10]. We performed immunohistochemistry (IHC) by first deparaffinizing the sections, following which we performed antigen retrieval at $121^{\circ} \mathrm{C}$ in an autoclave for $10 \mathrm{~min}$ in a $10 \mathrm{mM}$ sodium citrate buffer ( $\mathrm{pH} \mathrm{6.0).} \mathrm{We} \mathrm{then} \mathrm{treated} \mathrm{the}$ sections with a $3 \% \mathrm{H}_{2} \mathrm{O}_{2}$ solution (Envision Plus System; Dako, Santa Clara, CA, USA) to inhibit any endogenous peroxidases. Rabbit polyclonal LIF antibody (1: 500, ab113262; Abcam) was used for IHC. The labeled antigens were visualized by chromogen 3,30diaminobenzidine tetrahydrochloride; hematoxylin was used as a nuclear counterstain. Eventually, the slides were observed under a fluorescence microscope (BZ-710; Keyence).

\subsection{Metabolomic analysis}

Monocultured and co-cultured Capan-1 cells and AD-MSCs (each group n =3) and KPC xenograft mice tumors $(n=3)$ were analyzed by metabolomics (Human Metabolome Technologies (HMT) Inc., Tsuruoka, Japan) [21] [22] [23]. Firstly, frozen cells or mouse tumor samples were transferred into $500 \mu \mathrm{L}$ of methanol containing $50 \mathrm{mM}$ of an external standard. Thereafter, we homogenized the cells five times at $200 \mathrm{x} \mathrm{g}$ for $120 \mathrm{~s}$ by BMSM10N21 (BMS, Tokyo). Following this, we added and mixed $500 \mu \mathrm{L}$ of chloroform and $200 \mu \mathrm{L}$ of ultrapure water to the homogenate, and subsequently centrifuged the mixture at 2,300 $\times \mathrm{g}$ for $5 \mathrm{~min}$ at $4{ }^{\circ} \mathrm{C}$. The resultant aqueous phase was subjected to ultrafiltration using a Millipore Ultrafree-MC PLHCC HMT Centrifugal Filter Device, 5 kDa (Millipore, Billerica, MA). The filtrates were then dried and dissolved in $50 \mu \mathrm{L}$ of ultrapure water. Subsequently, the samples were subjected to capillary electrophoresis time-of-flight mass spectrometry (CE-TOFMS) in the Agilent CE-TOFMS system (Agilent Technologies, Santa Clara, CA) at $4{ }^{\circ} \mathrm{C}$. The detected peaks were aligned according to the $\mathrm{m} / \mathrm{z}$ values and normalized migration times. Furthermore, the peaks were mean-centered and scaled using their standard deviations on a per-peak basis as a pretreatment. Following the application of autoscaling, we conducted principal component analysis (PCA) and hierarchical clustering analysis using SampleStat ver.3.14 (HMT Inc.) and PeakStat ver.3.18 (HMT Inc.). In the PCA, score plots of the first and second principal components were generated. Additionally, we generated heat maps by coloring the data values across their value ranges. The relative area of each peak was calculated and used for comparison among the four groups. 


\subsection{RNA extraction and quantitative real-time PCR ( $q P C R$ )}

We extracted total RNA from the cells using the TRI Reagent (Molecular Research Center, Inc., Cincinnati, OH, USA), according to the manufacturer's instructions. Subsequently, $500 \mathrm{ng}$ of the extracted RNA was converted to cDNA using RevaTra Ace reverse-transcription reagents (TOYOBO, Osaka, Japan), as per the manufacturer's instructions. Next, we performed qPCR using commercially available gene-specific PrimeTime qPCR probes (listed below; purchased from Integrated DNA Technologies, Coralville, CA, USA) and 29 Thunderbird Probe qPCR mix (TOYOBO), in accordance with the manufacturer's instructions. The following PrimeTime qPCR probes were used (Hs, Human probes): C-X-C motif chemokine ligand 1 (CXCL1), Hs.PT.58.39039397; glyceraldehyde-3-phosphate dehydrogenase (GAPDH), Hs.PT.39a.22214836; actin alpha 2, smooth muscle (ACTA2), Hs.PT.56a.2542642; Interleukin 6 (IL6), Hs.PT.58.40226675; leukemia inhibitory factor (LIF), Hs.PT.58.27705899; and connective tissue growth factor (CTGF), Hs.PT.58.14485164.g. Expression levels of the target genes were normalized to that of GAPDH.

\subsection{RNA-sequencing (RNA-seq)}

We evaluated the difference in the gene expression levels of AD-MSCs and AD-CAFs by performing RNA-seq as previously reported [10] [18]. Briefly, total RNA was extracted from the cells using the TRI Reagent (Molecular Research Center, Inc., Cincinnati, $\mathrm{OH}$, USA). Thereafter, library preparation and sequencing were conducted using the Truseq library prep kit and NovaSeq 6000 (Illumina, San Diego, CA, USA). The data acquired from two biological replicates for each cell type were analyzed using STAR (2.7.1a) [24], RSEM (1.3.1) [25], and edgeR (3.30.3) [26]. Subsequently, we obtained the normalized counts (trimmed mean of $\mathrm{M}$ values) and identified the differentially expressed genes that satisfied the condition $\mid \log 2$ (fold-change) $\mid \geq 1$ and false discovery rate $<0.05$. Raw sequences in the FASTQ format were deposited at the DNA Data Bank of Japan (DDBJ; accession numbers DRR231745- DRR231748).

\subsection{Statistical analysis}

Data are represented as the mean \pm standard deviation (SD) unless otherwise noted. We analyzed the data among groups of three or more groups by one-way analysis of variance followed by post-hoc Tukey tests with two-tailed distribution. Furthermore, the student's t-test was used to compare data between the control and experimental groups. Statistical significance was set at $\mathrm{p}<0.05$. All calculations were performed using the GraphPad Prism software or EZR (Saitama Medical Center, Jichi Medical University, Saitama, Japan), which is a graphical user interface of the $R$ software ( $R$ Foundation for Statistical Computing) [27]. Of note, EZR is a modified version of R Commander designed for statistical functions that are frequently used in biostatistics. Additionally, the SD of the data are represented as error bars in the figures.

\section{Results}

3.1 AD-MSCs co-cultured with Capan-1 cells are CAF-progenitors that are capable of reacting with cancer cells

We generated CAFs derived from AD-MSCs (AD-CAFs) by two methods: direct contact and indirect non-contact transwell co-culture with the Capan-1 cell line. Our qPCR analysis confirmed that these two methods generated AD-CAFs by two distinct mechanisms of differentiation (Figure 1a). For instance, AD-CAFs generated by contact co-culture exhibited increased expression of the myoblast markers ACTA2 and CTGF. On the other hand, AD-CAFs generated by the non-contact co-culture had upregulated expression of cytokine-related genes, such as CXCL1, IL6, and LIF. These alterations were verified by immunostaining (Figure 1b). Moreover, the $\alpha$ SMA protein was more prominently expressed in the direct co-culture than in the non-contact co-culture. Notably, IL6-positive cells were rarely observed in this approach. In contrast, the number of IL6-positive cells was greatly increased and $\alpha$ SMA-positive cells were not observed 
when cells were cultured via the non-contact transwell co-culture. However, vimentin, a fibroblast marker, had uniform expression in both the co-cultures, confirming that the analyzed cells were indeed CAFs. Next, we examined whether co-culturing GFPtransfected AD-MSCs with RFP-transfected Capan-1 cells resulted in cell-cell interactions. Indeed, we observed vesicles after three days of contact co-culture (Figure 1c), and the presence of green vesicles in Capan-1 cells or red vesicles in CAFs was confirmed under a strong magnification (Figure 1d). Furthermore, we observed the presence of several FITC-positive vesicles when dextran-FITC was added to the co-culture media (Figure 1e). These observations verified that AD-MSCs differentiate into AD-CAFs when co-cultured with Capan-1 cells. Therefore, we used AD-MSCs as progenitors of CAFs for subsequent metabolomic analysis. Notably, we used AD-MSCs maintained in non-contact co-cultures in consideration of the time required for sample collection and to prevent contamination between AD-MSCs and Caplan-1 cells.

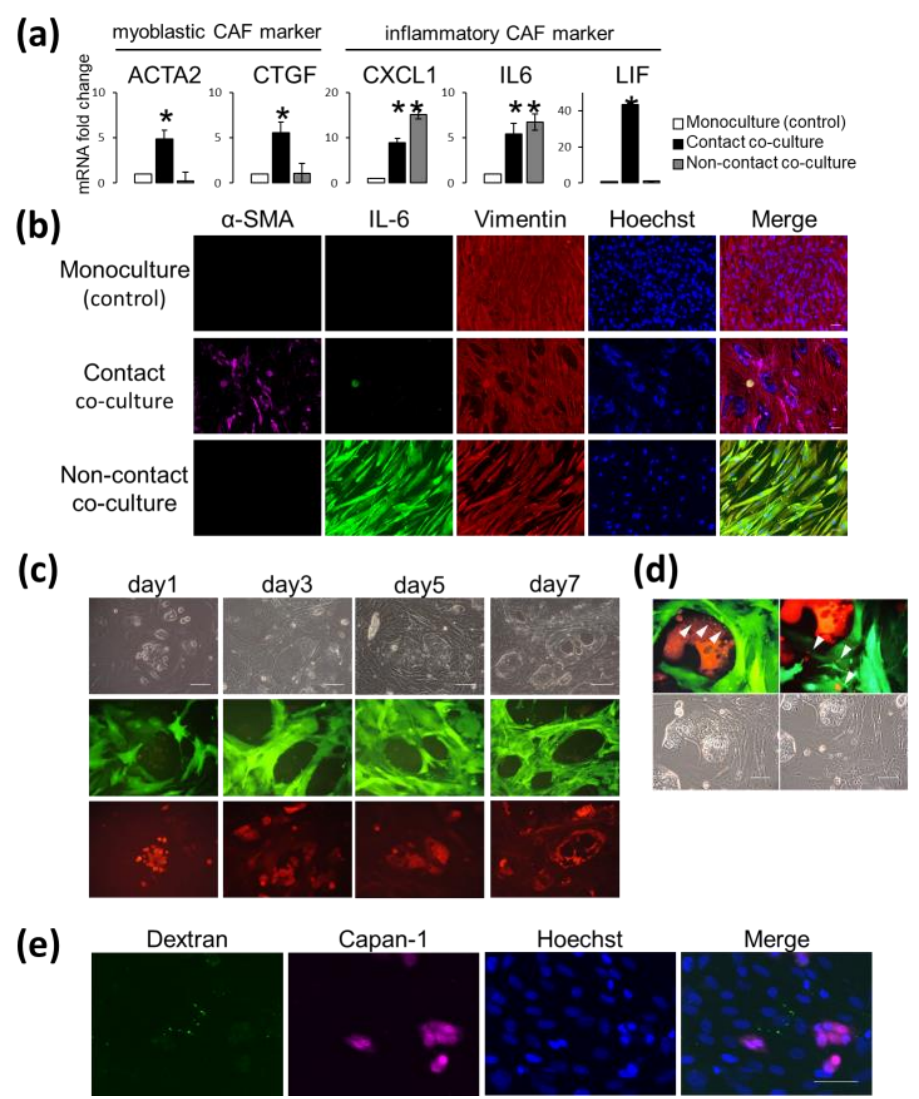

Figure 1. Adipose-derived mesenchymal stem cells (AD-MSCs) differentiated into adipose-derived cancer-associated fibroblasts (AD-CAFs) upon co-culture and interaction with Capan-1 cells. (a) qPCR analysis of myoblastic cancer-associated fibroblast (CAF) markers or inflammatory CAF markers in three culture conditions. Results are presented as the mean \pm standard deviation of three biological replicates. ${ }^{*} p<0.05$, unpaired Student's t-test. (b) Representative immunofluorescent image of AD-MSCs co-cultured with/without Capan-1 cells and stained for $\alpha$ SMA (magenta), IL- 6 (green), and vimentin (red). Counterstain: Hoechst 33342 (blue). Scale bar: $100 \mu \mathrm{m}$. (c) Representative image of direct contact co-culture of green fluorescent protein (GFP)-tagged CAFs with red fluorescent protein (RFP)-tagged Capan-1 cells on days 1, 3, 5, and 7. Scale bar: $100 \mu \mathrm{m}$. (d) Magnified images of direct contact co-culture of GFP-tagged CAFs with RFP-tagged Capan-1 cells on day 7. Arrowheads indicate vesicles formed by interaction of AD-MSCs with Capan-1 cells. Scale bar: $50 \mu \mathrm{m}$. (e) Representative fluorescent image confirming macropinocytosis in the co-culture of AD-MSCs and Capan-1 cells (magenta) after the addition of FITC-dextran. Counterstain: Hoechst 33342 (blue). Scale bar: $50 \mu \mathrm{m}$. 
3.2 AD-CAFs undergo a more drastic metabolic transformation than Capan-1, as discovered by a global metabolomic analysis

We performed metabolomic analyses of AD-MSCs (progenitors of CAFs), AD-CAFs, and Capan- 1 cells before and after co-culture by CE-TOFMS (Figure 2a). The results of our PCA are presented in Figure 2a. Remarkably, we observed that the metabolite profile of AD-MSCs was significantly different from AD-CAFs. The metabolites involved in the PC2-axis in Figure 2a, and their contribution rates are represented in Figure 2b. Interestingly, these include metabolites that are involved in polyamine metabolism, but not those directly involved in major intracellular metabolic pathways, such as glycolysis and OXPHOS. Since these identified metabolites have not been well-studied, they are possible novel biomarkers of CAF- abundant tumors; we have discussed this matter in detail in later sections. On the other hand, we observed only marginal metabolic changes in Capan-1 cells, indicating that these cells are stable as established cancer cells. A heat map of the metabolite changes in each cell type is shown in Figure 2c. Overall, metabolites that were abundant in Capan-1 cells were scarce in CAFs, and conversely, metabolites that were abundant in CAFs were scarce in Capan-1 cells. This held true for metabolites that were highly abundant in Capan-1 cells both before and after co-culturing but were almost absent in CAFs both before and after co-culturing. These results indicate that each cell type had its own specific metabolite profile. Remarkably, 36 metabolites exhibited altered abundance in Capan-1 cells; while 12 (33\%) were increased in number, $24(24 \%)$ were decreased. In contrast, 86 metabolites were altered in CAFs, of which $67(78 \%)$ were downregulated. Thus, the metabolite changes observed in CAFs were larger than those in observed in Capan-1 cells; notably, these changes indicated a decrease in abundance of the metabolites.

(a)

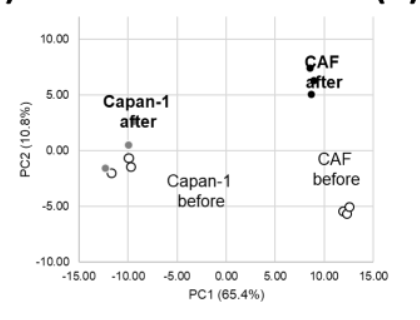

(c)

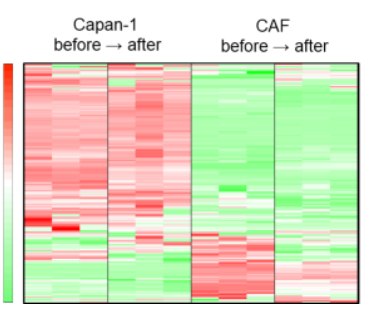

(b)

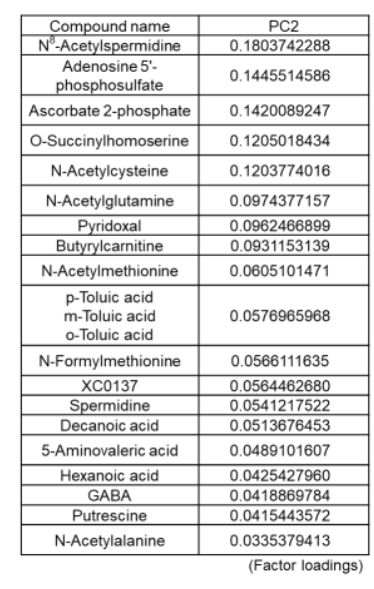

Figure 2. Global trend of metabolite changes in cancer cells (Capan-1 cells) and cancer-associated fibroblasts (CAFs). (a) Principal component analysis (PCA) of metabolomic datasets of Capan-1 cells and CAFs grown in a non-contact transwell co-culture. (b) The metabolites involved in PC2-axis and their contribution rates. (c) Heat map comparing altered metabolites between Capan-1 cells ( $\mathrm{n}$ $=3)$ and CAFs $(n=3)$.

\subsection{AD-CAFs exhibit upregulated glycolytic metabolism}

We examined the changes in intracellular metabolism by comparing the metabolite profiles between AD-CAFs and AD-MSCs. Figure 3a depicts changes in the glycolytic metabolites, including glucose. While the levels of glucose 6-phosphate were substantially decreased upon differentiation of AD-MSCs to AD-CAFs, those of fructose 1,6-phosphate and phosphoenolpyruvic acid were increased by 1.9 and 1.4 folds, respectively. In addition, the aerobic glycolysis metabolite lactate was significantly increased ( 1.5 fold) in AD-CAFs, indicating that these cells undergo a metabolic shift towards glycolysis. We further validated this metabolic shift by analyzing altered gene expression levels by RNA- 
seq. The list of genes involved in glycolysis and their expression levels are displayed in Figure 3b. Consequently, we identified HK2, PFKL, PFKP, ALDOC, GAPDH, PGK1, ENO1, $E N O 2$, and $L D H A$ as differentially expressed genes (i.e., upregulated or downregulated gene expression). However, our pathway analysis did not reveal any significant changes, and the metabolic shift to glycolysis was not supported by our gene expression analysis.

(a)

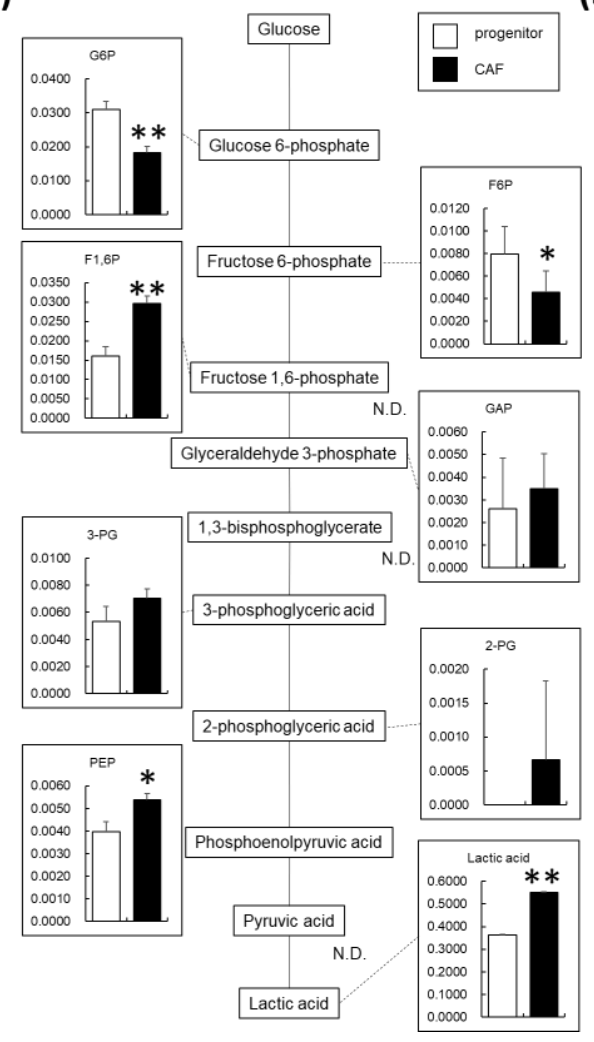

(b)

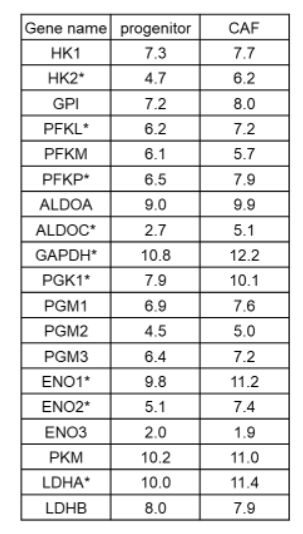

Figure 3. Changes in metabolites involved in glucose metabolism in the cancer-associated fibroblast (CAF) progenitors and CAFs. (a) Graphical representation of relative metabolite changes between CAF progenitors and CAFs as determined by capillary electrophoresis time-of-flight mass spectrometry. N.D. indicates not detected. ${ }^{* *} p<0.01,{ }^{*} p<0.05$ (b) List of genes involved in glycolysis and their expression levels in CAF progenitors and CAFs. Log2 normalized counts per million values. Asterisks $\left(^{*}\right)$ indicate differentially expressed genes.

\subsection{OXPHOS is downregulated in AD-CAFs}

Changes in the metabolites associated with the tricarboxylic acid (TCA) cycle are shown in Figure 4a. In this cycle, acetyl-CoA is synthesized from pyruvate and is converted to citric acid, and ATP is produced during reactions involving cis-aconitic acid, 2-oxoglutaric acid, and malic acid. Notably, acetyl-CoA (-7.4 fold), citric acid (-1.5 fold), and malic acid (-1.3 fold) were observed to be considerably downregulated in AD-CAFs. Indeed, we determined that downregulation of OXPHOS was associated with upregulation of glycolysis in AD-CAFs. The list of genes involved in OXPHOS and their expression levels are presented in Figure $4 \mathrm{~b}$. Remarkably, we observed no significant changes in the mRNA expression levels of these genes. Thus, the metabolic shift towards glycolysis in the AD-CAFs was not verified by changes in the gene expression levels. 
(a)

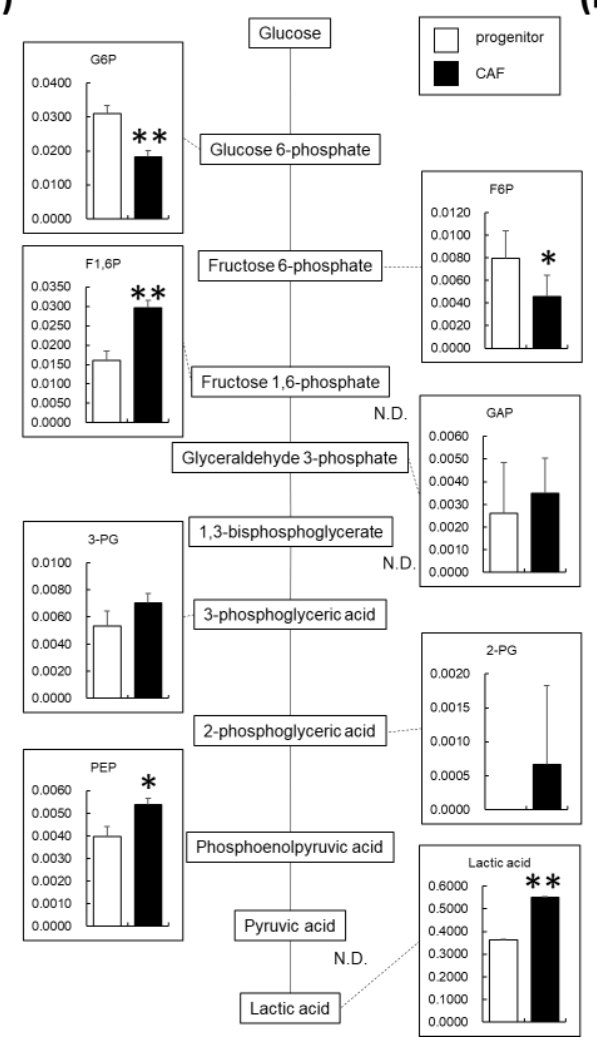

(b)

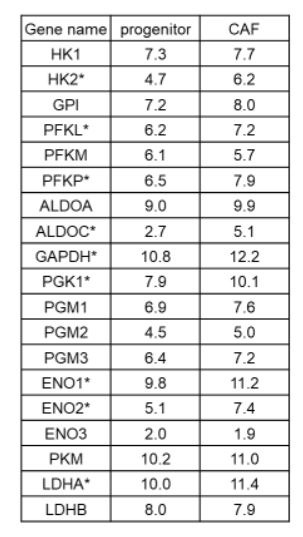

Figure 4. Changes in metabolites related to the tricarboxylic acid (TCA) cycle in the cancerassociated fibroblast (CAF) progenitors and CAFs. (a) Graphical representation of relative metabolite changes between CAF progenitors and CAFs, as determined by capillary electrophoresis time-of-flight mass spectrometry. N.D. indicates not detected. ${ }^{* *} p<0.01,{ }^{*} p<0.05$ (b) List of genes involved in the TCA cycle and their expression levels. Log2 normalized counts per million values. Asterisks $\left({ }^{*}\right)$ indicate differentially expressed genes.

\subsection{Polyamine metabolism is altered in AD-CAFs.}

Polyamines are involved in various physiological functions, such as cell division and proliferation and nucleic acid and protein synthesis; studies have also determined their roles in cancer cells [28-30]. Since we identified several polyamine metabolites in the PC2 axis in the PCA analysis (Figure 1), we also analyzed the polyamine synthesis pathway. Figure 5a presents changes in ornithine synthesized from arginine and those observed in metabolites involved in the polyamine synthesis pathway. We observed that ornithine, the starting point of this pathway, was significantly downregulated by 1.3 fold in the ADCAFs. Moreover, while putrescine, N8-acetylspermidine, N-acetylputrescine, and 5'deoxy-5'-methylthioadenosine were not detected in AD-MSCs, they were detected in ADCAFs. A list of genes involved in the polyamine synthesis pathway and their expression levels are listed in Figure $5 \mathrm{~b}$. Since we observed no significant changes in the gene expression levels, the metabolic shift towards glycolysis was not supported by our gene expression analysis. 
(a)

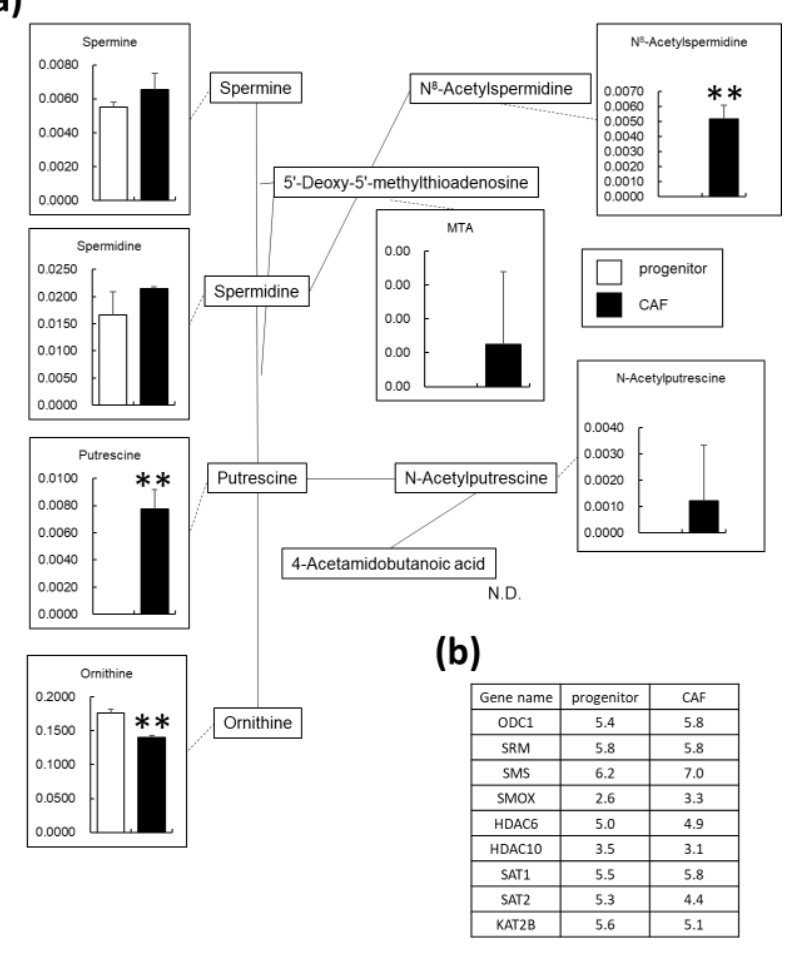

Figure 5. Changes in metabolites related to polyamine metabolism in cancer-associated fibroblasts (CAF) progenitors and CAFs. (a) Graphical representation of relative metabolite changes between CAF progenitors and CAFs, as determined by capillary electrophoresis time-of-flight mass spectrometry. N.D. indicates not detected. ${ }^{* *} p<0.01$. (b) List of genes involved in polyamine metabolism and their expression levels. Log2 normalized counts per million values. There were no differentially expressed genes.

\subsection{Certain metabolites are uniquely present in $A D-C A F s$}

Spermidine is acetylated at the $\mathrm{N} 8$ position by $\mathrm{N}$-acetyltransferase in the cell nucleus. Subsequently, it is transported to the cytoplasm, where it is deacetylated by metaldependent N8-acetylspermidine deacetylase, also called polyamine deacetylase, and N8acetylspermidine is synthesized [31,32]. Alterations in the levels of N8-acetylspermidine in all the samples, including Capan-1 cells, are presented in Figure 6a. Interestingly, we detected this metabolite only in AD-CAFs. Therefore, we deduced that it is possibly a specific biomarker for CAF-rich cancers. On the other hand, the metabolites that were not detected in CAF progenitors but were detected in AD-CAF, although they were not changed in Capan-1 before and after co-culture are shown in Figure 6b. One such metabolite was the polyamine putrescine. Indeed, putrescine was detected in both ADCAF and Capan-1. However, considering that tumors in vivo are a mixture of cancer cells and CAFs, the total amount of putrescine was increased after co-culture. Therefore, putrescine may be a specific biomarker. Similarly, N-acetylcysteine, Osuccinylhomoserine, and butyrylcarnitine could be regarded as specific metabolites of pancreatic cancer, as they were detected specifically in AD-CAFs. 
(a)

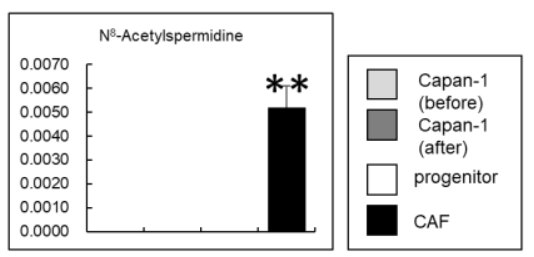

(b)
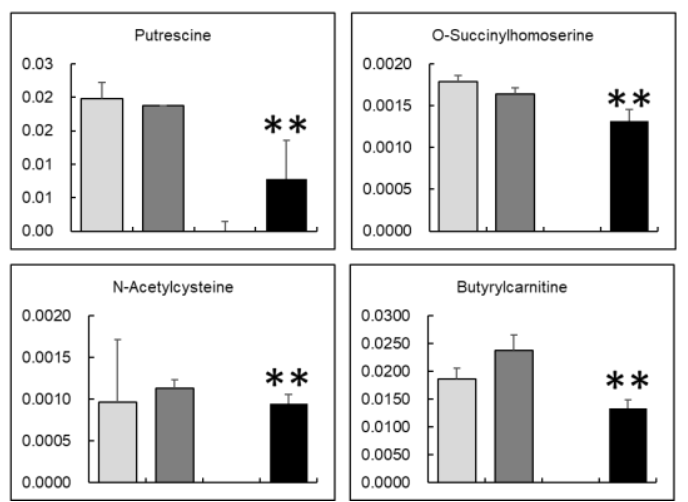

Figure 6. Metabolites specifically detected in cancer-associated fibroblasts (CAFs) alone and not CAF progenitors. (a) Amount of N8-acetylspermidine in all samples. N8-Acetylspermidine was detected only in CAFs. ${ }^{* *} p<0.01$. (b) Four metabolites detected solely in CAFs and not in the progenitors. ${ }^{* *} p<0.01$.

\subsection{Metabolites unique to AD-CAFs are also expressed in mouse pancreatic cancer models}

Next, we examined whether these AD-CAF-specific metabolites could also be detected in mouse tumor tissues (Figure 7a). Figure 7b displays the tumor tissue section. In this mouse model, mouse cells were spontaneously induced to differentiate into CAFs, as confirmed by the MT staining. This model also contained a population of LIF-positive CAFs. Tumor samples were collected from three independent mice, and metabolomic analyses were performed (Figure 7c, d). N8-Acetylspermidine was indeed detected, as in the in vivo analysis, and other metabolites were also detected in living tumor tissues; however, some of these metabolites were close to the detection limit. 


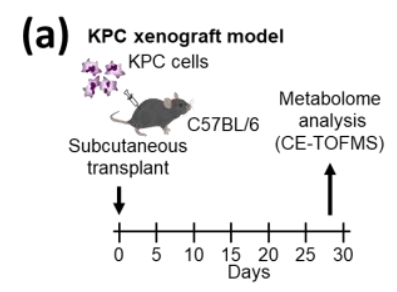

(b)

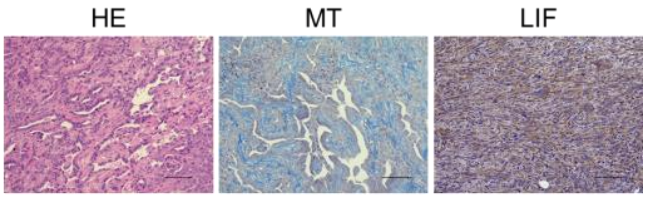

(c)

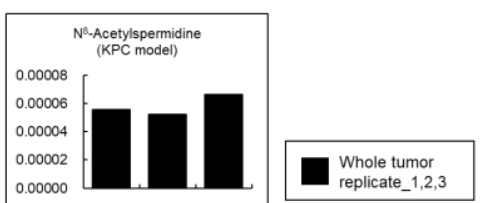

(d)
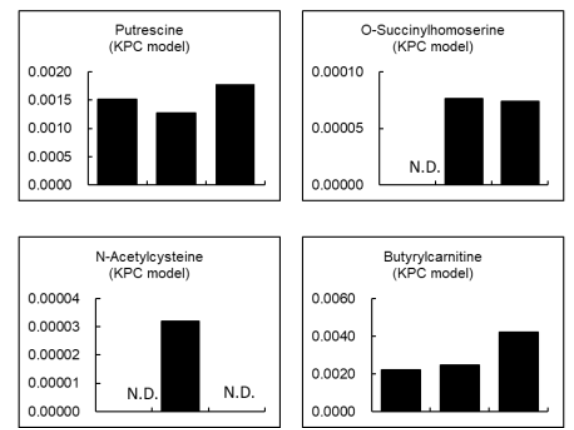

Figure 7. Unique metabolites detected in adipose-derived cancer-associated fibroblasts (AD-CAFs) in vitro were also detectable in mouse pancreatic cancer models. (a) Schematic illustration of the KPC xenograft model and treatment protocol. KPC cells were transplanted subcutaneously into C57BL/6 mice, and excised tumors were subjected to metabolomic analysis. (b) Representative images of the KPC xenograft tumor. These tumors recapitulate the human pancreatic cancer. Scale bar: $100 \mu \mathrm{m}$. (c) N8-Acetylspermidine was detected in the KPC xenograft tumor. (d) Four metabolites detected solely in the AD-CAFs and not in the progenitors were also detected in the KPC xenograft tumor.

\section{Discussion}

\subsection{Metabolic transformation in CAFs}

In this study, we used an original experimental approach to induce cell differentiation into CAFs in vitro and performed metabolomic analysis to distinguish cancer cells from CAFs. Since the TME contains CAFs in addition with various cell types, such as blood or immune cells, it is difficult to understand the metabolic changes that occur in each cell type. However, in this study, we accurately determined the metabolic transformations that occur in CAFs. Previously, molecular analyses have revealed changes in metabolic genes expressed in CAFs [33-35]. These results suggested that CAFs within tumors have an active glycolytic metabolism. In this study, we verified AD-MSCs as the progenitors of CAFs. Furthermore, we compared AD-MSCs and AD-CAFs to precisely elucidate the metabolic changes that occur during differentiation of AD-MSCs into CAFs. The cancer cell line used in this study was a human pancreatic cancer cell line. However, the robustness and homology of this data needs to be verified in various types of cancer cells, including those derived from various solid tumors, such as gastric cancer and colon cancer. 


\subsection{Polyamine etabolism in CAFs}

Putrescine and N8-acetylspermidine were detected as metabolites specific to CAFs. Putrescine is synthesized from ornithine and is converted to spermidine. In turn, spermidine is converted to N8-acetylspermidine by the histone acetyltransferase P/CAF (KAT2B) in the presence of acetyl CoA. Since the synthesized N8-acetylspermidine is deacetylated by polyamine deacetylase, decreased activity of this enzyme causes accumulation of N8-acetylspermidine in CAFs. However, owing to the high substrate specificity of polyamine deacetylase, it does not deacetylate other acetylspermidines, such as cytoplasmic N1-acetylspermidine or N1-acetylspermine [36]. In addition, selective inhibition of polyamine deacetylase activity in HeLa cells has been demonstrated to increase N8-acetylspermidine levels but not acetylated histone levels. Additionally, polyamine deacetylase has a different function than histone diacetylase (HDAC) [37]. Recently, studies have reported that two $\mathrm{Zn}^{2+}$-dependent HDACs, HDAC6 and HDAC10, function as polyamine deacetylases $[38,39]$. Therefore, we also examined the expression of HDAC6 and HDAC10 in AD-MSCs and AD-CAFs but found no changes in their mRNA levels (data not shown). However, to investigate whether N8-acetylspermidine accumulates in CAFs because of decreased activity of polyamine deacetylase, it is necessary to measure the amount and activity of the enzyme products. Notably, putrescine was upregulated in AD-CAFs, suggesting that N8-acetylspermidine may have been synthesized more by $\mathrm{P} / \mathrm{CAF}$ via spermidine in the CAFs than progenitors. However, we observed no changes in the mRNA levels of KAT2B or HDAC (data not shown). Further studies are needed to determine the pathway of N8-acetylspermidine synthesis and metabolism by performing detailed enzyme activity and metabolite flux analyses.

\subsection{N8-Acetylspermidine is a potential biomarker of pancreatic cancer}

N8-Acetylspermidine was the only AD-CAF-specific metabolite identified in this study. Since this metabolite was not detected in pancreatic cancer cells, its levels may be an indicator of the amount of CAFs present in tumors. Furthermore, we detected this metabolite in a pancreatic cancer mouse model. Since N8-acetylspermidine was not detected in Capan-1 cells, we assumed that N8-acetylspermidine is only biosynthesized in CAFs in vivo. Remarkably, N8-acetylspermidine has been detected more frequently in patients with early-stage pancreatic cancer than in that in healthy subjects, and thus is likely to be a promising CAF-specific metabolite [40]. The other metabolites presented in Figure 6 are either biosynthesized in CAFs or taken up by CAFs from Capan-1 cells. As we observed macropinocytosis and secretion of extracellular vesicles, such as exosomes, in the TME, and we determined that putrescine is synthesized in Capan-1 cells and CAFs, suggesting that it may be incorporated into CAFs via intercellular transport mechanisms. Nonetheless, these observations need further verifications.

\section{Conclusions}

In this study, we used a simple in vitro experimental system to analyze metabolites with high accuracy and identify several factors that could be potential biomarkers of pancreatic cancer. Although the CAF types produced in this simple experimental system may be limited, the potential biomarkers identified in this study are expected to prove to be important in pancreatic cancer in vivo.

Author Contributions: YM and YSK conceived the study. YM performed the experiments. YM, NM, YA and YSK analyzed the data. TO and YSK supervised the experiments. YM and YSK wrote the manuscript. All authors reviewed and approved the final manuscript draft for submission.

Funding: This work was partially supported by JSPS KAKENHI (grant numbers JP15H04924 and JP25670567) and AMED (grant number JP18be0304401j0002). This work was also supported by the Astellas Foundation for Research on Metabolic Disorders Foundation.

Institutional Review Board Statement: All animal experiments and procedures were approved by the Institutional Animal Care and Use Committee of the respective National Institute of Advanced Industrial Science and Technology (A2020-310) and the Ethics Committee of the University of 
Tsukuba (19-028) and were carried out in accordance with the approved guidelines. The study was conducted in compliance with the Animal Research Reporting in vivo Experiments (ARRIVE) guidelines [20].

Data Availability Statement: RNA sequencing data is available at the DDBJ. Read Archive under accession number DRR231745- DRR231748. Raw data are available from the corresponding author upon request.

\begin{abstract}
Acknowledgments: The authors would like to thank the members of the Cellular and Molecular Biotechnology Research Institute and GI\&HBP Surgery of Tsukuba for their input and helpful comments. The authors are also grateful to Chiaki Okamoto for their technical assistance and Yasuko Ozaki and Tomoko Ataka for their secretarial assistance. The KPC cell line derived from KPC mice was a kind gift from Eric O' Neill (Oxford University). Computational analyses were partially performed on the NIG supercomputer at the ROIS National Institute of Genetics. The images of mice were obtained from TogoTV (C2016 DBCLS TogoTV / CC-BY-4.0). We would like to thank Editage (www.editage.com) for English language editing.
\end{abstract}

Conflicts of Interest: The authors declare no conflict of interest.

\title{
References
}

1. Tjomsland, V.; Niklasson, L.; Sandstrom, P.; Borch, K.; Druid, H.; Bratthall, C.; Messmer, D.; Larsson, M.; Spangeus, A. The desmoplastic stroma plays an essential role in the accumulation and modulation of infiltrated immune cells in pancreatic adenocarcinoma. Clinical \& developmental immunology 2011, 2011, 212810, doi:10.1155/2011/212810.

2. Liang, C.; Shi, S.; Meng, Q.; Liang, D.; Ji, S.; Zhang, B.; Qin, Y.; Xu, J.; Ni, Q.; Yu, X. Complex roles of the stroma in the intrinsic resistance to gemcitabine in pancreatic cancer: where we are and where we are going. Experimental \& Molecular Medicine 2017, 49, e406-e406, doi:10.1038/emm.2017.255.

3. Neesse, A.; Bauer, C.A.; Öhlund, D.; Lauth, M.; Buchholz, M.; Michl, P.; Tuveson, D.A.; Gress, T.M. Stromal biology and therapy in pancreatic cancer: ready for clinical translation? Gut 2019, 68, 159-171, doi:10.1136/gutjnl-2018-316451.

4. Kalluri, R. The biology and function of fibroblasts in cancer. Nature reviews. Cancer 2016, 16, 582-598, doi:10.1038/nrc.2016.73.

5. Sahai, E.; Astsaturov, I.; Cukierman, E.; DeNardo, D.G.; Egeblad, M.; Evans, R.M.; Fearon, D.; Greten, F.R.; Hingorani, S.R.; Hunter, T.; et al. A framework for advancing our understanding of cancer-associated fibroblasts. Nature reviews. Cancer 2020, 10.1038/s41568-41019-40238-41561, doi:10.1038/s41568-019-0238-1.

6. Moir, J.A.; Mann, J.; White, S.A. The role of pancreatic stellate cells in pancreatic cancer. Surgical oncology 2015, 24, 232-238, doi:10.1016/j.suronc.2015.05.002.

7. Ohlund, D.; Handly-Santana, A.; Biffi, G.; Elyada, E.; Almeida, A.S.; Ponz-Sarvise, M.; Corbo, V.; Oni, T.E.; Hearn, S.A.; Lee, E.J.; et al. Distinct populations of inflammatory fibroblasts and myofibroblasts in pancreatic cancer. The Journal of experimental medicine 2017, 214, 579-596, doi:10.1084/jem.20162024.

8. Quante, M.; Tu, S.P.; Tomita, H.; Gonda, T.; Wang, S.S.; Takashi, S.; Baik, G.H.; Shibata, W.; Diprete, B.; Betz, K.S.; et al. Bone marrow-derived myofibroblasts contribute to the mesenchymal stem cell niche and promote tumor growth. Cancer cell 2011, 19, 257-272, doi:10.1016/j.ccr.2011.01.020.

9. $\quad$ Bochet, L.; Lehuede, C.; Dauvillier, S.; Wang, Y.Y.; Dirat, B.; Laurent, V.; Dray, C.; Guiet, R.; Maridonneau-Parini, I.; Le Gonidec, S.; et al. Adipocyte-derived fibroblasts promote tumor progression and contribute to the desmoplastic reaction in breast cancer. Cancer research 2013, 73, 5657-5668, doi:10.1158/0008-5472.Can-13-0530. 
10. Miyazaki, Y.; Oda, T.; Inagaki, Y.; Kushige, H.; Saito, Y.; Mori, N.; Takayama, Y.; Kumagai, Y.; Mitsuyama, T.; Kida, Y.S. Adipose-derived mesenchymal stem cells differentiate into heterogeneous cancer-associated fibroblasts in a stromarich xenograft model. Scientific reports 2021, 11, 4690, doi:10.1038/s41598-021-84058-3.

11. Elyada, E.; Bolisetty, M.; Laise, P.; Flynn, W.F.; Courtois, E.T.; Burkhart, R.A.; Teinor, J.A.; Belleau, P.; Biffi, G.; Lucito, M.S.; et al. Cross-Species Single-Cell Analysis of Pancreatic Ductal Adenocarcinoma Reveals Antigen-Presenting CancerAssociated Fibroblasts. Cancer discovery 2019, 9, 1102-1123, doi:10.1158/2159-8290.Cd-19-0094.

12. Dominguez, C.X.; Müller, S.; Keerthivasan, S.; Koeppen, H.; Hung, J.; Gierke, S.; Breart, B.; Foreman, O.; Bainbridge, T.W.; Castiglioni, A.; et al. Single-Cell RNA Sequencing Reveals Stromal Evolution into LRRC $15<\sup >+</$ sup $>$ Myofibroblasts as a Determinant of Patient Response to Cancer Immunotherapy. Cancer discovery 2020, 10, 232-253, doi:10.1158/2159-8290.Cd-19-0644.

13. Lee, H.; Zandkarimi, F.; Zhang, Y.; Meena, J.K.; Kim, J.; Zhuang, L.; Tyagi, S.; Ma, L.; Westbrook, T.F.; Steinberg, G.R.; et al. Energy-stress-mediated AMPK activation inhibits ferroptosis. Nat Cell Biol 2020, 22, 225-234, doi:10.1038/s41556020-0461-8.

14. Qin, C.; Yang, G.; Yang, J.; Ren, B.; Wang, H.; Chen, G.; Zhao, F.; You, L.; Wang, W.; Zhao, Y. Metabolism of pancreatic cancer: paving the way to better anticancer strategies. Molecular cancer 2020, 19, 50, doi:10.1186/s12943-020-01169-7.

15. Jones, C.L.; Inguva, A.; Jordan, C.T. Targeting Energy Metabolism in Cancer Stem Cells: Progress and Challenges in Leukemia and Solid Tumors. Cell stem cell 2021, 28, 378-393, doi:10.1016/j.stem.2021.02.013.

16. Li, X.; Zhu, H.; Sun, W.; Yang, X.; Nie, Q.; Fang, X. Role of glutamine and its metabolite ammonia in crosstalk of cancerassociated fibroblasts and cancer cells. Cancer cell international 2021, 21, 479, doi:10.1186/s12935-021-02121-5.

17. Li, Z.; Sun, C.; Qin, Z. Metabolic reprogramming of cancer-associated fibroblasts and its effect on cancer cell reprogramming. Theranostics 2021, 11, 8322-8336, doi:10.7150/thno.62378.

18. Miyazaki, Y.; Oda, T.; Mori, N.; Kida, Y.S. Adipose-derived mesenchymal stem cells differentiate into pancreatic cancerassociated fibroblasts in vitro. FEBS open bio 2020, 10, 2268-2281, doi:10.1002/2211-5463.12976.

19. Zhang, Y.; Recouvreux, M.V.; Jung, M.; Galenkamp, K.M.O.; Li, Y.; Zagnitko, O.; Scott, D.A.; Lowy, A.M.; Commisso, C. Macropinocytosis in Cancer-Associated Fibroblasts is Dependent on CaMKK2/ARHGEF2 Signaling and Functions to Support Tumor and Stromal Cell Fitness. Cancer discovery 2021, doi:10.1158/2159-8290.CD-20-0119.

20. Percie du Sert, N.; Ahluwalia, A.; Alam, S.; Avey, M.T.; Baker, M.; Browne, W.J.; Clark, A.; Cuthill, I.C.; Dirnagl, U.; Emerson, M.; et al. Reporting animal research: Explanation and elaboration for the ARRIVE guidelines 2.0. PLoS Biol 2020, 18, e3000411, doi:10.1371/journal.pbio.3000411.

21. Soga, T.; Heiger, D.N. Amino acid analysis by capillary electrophoresis electrospray ionization mass spectrometry. Analytical chemistry 2000, 72, 1236-1241, doi:10.1021/ac990976y.

22. Soga, T. Simultaneous Determination of Anionic Intermediates for Bacillus subtilis Metabolic Pathways by Capillary Electrophoresis Electrospray Ionization Mass Spectrometry. 2002.

23. Soga, T.; Kakazu, Y.; Robert, M.; Tomita, M.; Nishioka, T. Qualitative and quantitative analysis of amino acids by capillary electrophoresis-electrospray ionization-tandem mass spectrometry. Electrophoresis 2004, 25, 1964-1972, doi:10.1002/elps.200305791.

24. Dobin, A.; Davis, C.A.; Schlesinger, F.; Drenkow, J.; Zaleski, C.; Jha, S.; Batut, P.; Chaisson, M.; Gingeras, T.R. STAR: ultrafast universal RNA-seq aligner. Bioinformatics (Oxford, England) 2013, 29, 15-21, doi:10.1093/bioinformatics/bts635. 
25. Li, B.; Dewey, C.N. RSEM: accurate transcript quantification from RNA-Seq data with or without a reference genome. BMC bioinformatics 2011, 12, 323, doi:10.1186/1471-2105-12-323.

26. Robinson, M.D.; McCarthy, D.J.; Smyth, G.K. edgeR: a Bioconductor package for differential expression analysis of digital gene expression data. Bioinformatics (Oxford, England) 2010, 26, 139-140, doi:10.1093/bioinformatics/btp616.

27. Kanda, Y. Investigation of the freely available easy-to-use software 'EZR' for medical statistics. Bone Marrow Transplant 2013, 48, 452-458, doi:10.1038/bmt.2012.244.

28. Phanstiel, O.t. An overview of polyamine metabolism in pancreatic ductal adenocarcinoma. International journal of cancer 2018, 142, 1968-1976, doi:10.1002/ijc.31155.

29. Lieu, E.L.; Nguyen, T.; Rhyne, S.; Kim, J. Amino acids in cancer. Exp Mol Med 2020, 52, 15-30, doi:10.1038/s12276020-0375-3.

30. Nakkina, S.P.; Gitto, S.B.; Pandey, V.; Parikh, J.G.; Geerts, D.; Maurer, H.C.; Olive, K.P.; Phanstiel, O.t.; Altomare, D.A. Differential Expression of Polyamine Pathways in Human Pancreatic Tumor Progression and Effects of Polyamine Blockade on Tumor Microenvironment. Cancers 2021, 13, doi:10.3390/cancers13246391.

31. Michael, A.J. Biosynthesis of polyamines and polyamine-containing molecules. Biochem J 2016, 473, 2315-2329, doi:10.1042/bcj20160185.

32. Kahana, C. Protein degradation, the main hub in the regulation of cellular polyamines. Biochem J 2016, 473, 4551-4558, doi:10.1042/bcj20160519c.

33. Sung, J.S.; Kang, C.W.; Kang, S.; Jang, Y.; Chae, Y.C.; Kim, B.G.; Cho, N.H. ITGB4-mediated metabolic reprogramming of cancer-associated fibroblasts. Oncogene 2020, 39, 664-676, doi:10.1038/s41388-019-1014-0.

34. Becker, L.M.; O'Connell, J.T.; Vo, A.P.; Cain, M.P.; Tampe, D.; Bizarro, L.; Sugimoto, H.; McGow, A.K.; Asara, J.M.; Lovisa, S.; et al. Epigenetic Reprogramming of Cancer-Associated Fibroblasts Deregulates Glucose Metabolism and Facilitates Progression of Breast Cancer. Cell reports 2020, 31, 107701, doi:10.1016/j.celrep.2020.107701.

35. Wang, Y.; Liang, Y.; Xu, H.; Zhang, X.; Mao, T.; Cui, J.; Yao, J.; Wang, Y.; Jiao, F.; Xiao, X.; et al. Single-cell analysis of pancreatic ductal adenocarcinoma identifies a novel fibroblast subtype associated with poor prognosis but better immunotherapy response. Cell Discov 2021, 7, 36, doi:10.1038/s41421-021-00271-4.

36. Herbst-Gervasoni, C.J.; Christianson, D.W. Binding of N(8)-Acetylspermidine Analogues to Histone Deacetylase 10 Reveals Molecular Strategies for Blocking Polyamine Deacetylation. Biochemistry 2019, 58, 4957-4969, doi:10.1021/acs.biochem.9b00906.

37. Marchant, P.; Dredar, S.; Manneh, V.; Alshabanah, O.; Matthews, H.; Fries, D.; Blankenship, J. A selective inhibitor of N8-acetylspermidine deacetylation in mice and HeLa cells without effects on histone deacetylation. Archives of biochemistry and biophysics 1989, 273, 128-136, doi:10.1016/0003-9861(89)90170-7.

38. Hai, Y.; Shinsky, S.A.; Porter, N.J.; Christianson, D.W. Histone deacetylase 10 structure and molecular function as a polyamine deacetylase. Nature communications 2017, 8, 15368, doi:10.1038/ncomms15368.

39. Herbst-Gervasoni, C.J.; Steimbach, R.R.; Morgen, M.; Miller, A.K.; Christianson, D.W. Structural Basis for the Selective Inhibition of HDAC10, the Cytosolic Polyamine Deacetylase. ACS chemical biology 2020, 15, 2154-2163, doi:10.1021/acschembio.0c00362.

40. Fahrmann, J.F.; Bantis, L.E.; Capello, M.; Scelo, G.; Dennison, J.B.; Patel, N.; Murage, E.; Vykoukal, J.; Kundnani, D.L.; Foretova, L.; et al. A Plasma-Derived Protein-Metabolite Multiplexed Panel for Early-Stage Pancreatic Cancer. JNCI: Journal of the National Cancer Institute 2018, 111, 372-379, doi:10.1093/jnci/djy126. 
